\title{
Masking effectiveness and number of segments in the masking ring*
}

\author{
DONNA ARAND and WILLIAM N. DEMBER $\div$ \\ University of Cincinnati. Cincinnati. Ohio 45221
}

\begin{abstract}
The present study confirms an earlier finding, based on a spatial forced-choice detection task, that masking rings comprising alternating black and white segments are increasingly effective in the backward masking of a target disk as the number of those segments increases. However, as expected, that relation reached an asymptote for rings having 16 and 32 black segments; a complete, inverted V-shaped relation was not found, probably because the number of segments used was not sufficiently large.
\end{abstract}

The present study is one of a set of experiments on visual backward masking that applies forced-choice detection methodology to the disk-ring paradigm introduced by Werner (1935). In particular, it follows up two previous investigations on the masking effectiveness of incomplete masking rings. Sherrick \& Dember (1968) used masking stimuli having $40 \%$ of the contour of a complete black ring: this contour was distributed in a ring-shaped pattern. into 2. 4. 6, or 8 equal-sized black segments (alternated with an equal number of white segments). The masking effectiveness of these incomplete rings. as well as that of a complete ring and a no-ring mask. was assessed by measuring the detectability of a black disk when followed by the various masking stimuli. The data showed that masking effectiveness increased as number of ring segments increased.

In another related experiment (Dember, Colina, \& Sherrick. 1972). $25 \%, 50 \%$, or $75 \%$ of a complete black ring was removed: the remaining contour was symmetrically distributed in a ring-shaped pattern into four equal-sized segments. A second set of masking rings was also used. This set consisted of rings which varied in figure-ground contrast so as to yield complete rings having $25 \%$. $50 \%$ or $75 \%$ of the luminance of the white background on which the stimulus figures were presented. Thus, for each degree of ring-incompleteness in the one set. there was a contrast-reduced gray ring in the other set. In this case. it was found that each gray ring was less effective as a mask than was its incomplete counterpart.

The purpose of the present experiment was to test the hypothesis that the relationship found by Sherrick and Dember (1968) would reach asymptote at some value of number of ring segments greater than 8 , where, we assumed. the limits of visual acuity would transform a physically incomplete ring into one that functioned like a complete, but contrast-reduced, and hence (according

*This research was supported by Grant GB-33621 from The National Science Foundation to William N. Dember.

tR equests for reprints should be sent to William N. Dember, Department of Psychology. University of Cincinnati, Cincinnati, Ohio 45221. to Dember. Sherrick, \& Colina, 1972) less effective gray ring. Beyond that value, we expected masking effectiveness to decline with increasing number of ring segments. In short, we hypothesized that an inverted V-shaped relation between number of ring segments and masking effectiveness would be found if a sufficiently large number of segments was employed.

\section{METHOD}

\section{Subjects}

Four male and four female paid college students with normal or corrected to normal vision served as Ss. The Ss were trained on all stimuli used in the experiment, receiving 200 trials on each of 2 days before collection of experimental data.

\section{Stimuli}

The stimuli were first constructed using black and white drawing paper and then reduced photographically. The target stimulus was alway's a solid black disk, while the masking ring varied. Rings with 2. 4, 6, 8, 16, and 32 black segments were used. the total black coutour of all rings being $40 \%$ of that of the complete ring. In addition, a complete black ring and a blank white card were used in the masking condition.

The stimuli were cut from the photographic prints and glued on white paper. The diameter of the disk as well as the inner diameter of the rings measured $8 \mathrm{~mm}$; the outer diameter of the rings measured $16 \mathrm{~mm}$ : the distance between the centers of each pair of rings was $21 \mathrm{~mm}$ : these values correspond to $23.5 \mathrm{~min}$. $47 \mathrm{~min}$, and $1 \mathrm{deg}, 19 \mathrm{~min}$ of arc, respectively. With the exception of the blank card, each masking stimulus actually consisted of a pair of identical rings in order to accomodate the spatial forced-choice procedure. Also, the two-segment mask took two forms. One with gaps at the 12 o'clock and 6 o'clock positions and another with gaps at the $30^{\circ}$ clock and 9 o'clock positions. Each $\mathrm{S}$ received two sets of trials with each form during the experiment.

\section{Apparatus and Procedure}

Stimuli were presented tachistoscopically (Scientific Prototype, Model GX). The target and mask durations were 15 and $55 \mathrm{msec}$. respectively. There was no interstimulus interval. The luminance of the target, mask, and pre- and postadapting fields was $25 \mathrm{fL}$. View ing was monocular.

Each $\mathrm{S}$ experienced each of the eight mask conditions, in a block of 25 trials. during each of the four test sessions. At the completion of the experiment. each S had run 100 trials under each condition. To control for sequence effects. the order of presentation of the mask conditions was randomized for each $\mathrm{S}$. 
From trial to trial. E placed the disk so that it fit snugly within one of the two rings, the specific disk location being random. In the no-mask condition. the disk was also randomly placed to the right or left side. After each sequence of disk and mask. S indicated where the disk had appeared by say ing "right" or "left." guessing if necessary.

\section{RESULTS AND DISCUSSION}

Before analysis, data from each $\mathrm{S}$ was corrected for chance guessing. Mean percent correct detection took the following values: no ring, $100 \%: 2$ segments, $66.25 \%$; 4 segments, $56.5 \%$ : 6 segments, $48 \% ; 8$ segments, $43 \%$; 16 segments, $29.75 \%$ : 32 segments, $31.5 \%$; complete ring, $35.5 \%$. An analysis of variance on the data yielded a significant effect of mask condition $(F=10.21$, $\mathrm{p}<.001)$ and $\mathrm{Ss}(\mathrm{F}=10.82, \mathrm{p}<.001)$.

The data show the positive relation between maskability and the number of segments in the masking ring previously reported by Sherrick \& Dember (1968); furthermore, as expected, the relation does reach asymptote between 8 and 16 black segments. However, between the values of 16 and 32 segments. target detectability does not appreciably increase. Apparently. the number of ring segments used was not large enough to effect the complete inverted V-relationship that was hypothesized. Evidence for such a relationship awaits further experimentation with masks containing a greater number of segments than those used in the present study.

\section{REFERENCES}

Dember, W. N., Colina, T. L., \& Sherrick, M. F. Amount vs contrast of masking-figure contour in visual backward masking. Psychonomic Science, 1972, 27, 95-96.

Sherrick, M. F., \& Dember, W. N. Configurational factors in visual backward masking. Proceedings of the 76 th Annual Convention, American Psychological Association, 1968, 3, 111-112.

Werner, H. Studies on contour I: Qualitative analysis. American Journal of Psychology, 1935, 47, 40-64.

(Received for publication November 16.1973.) 